Mr. S. E. Peal, I may, therefore, be communicating stale infor mation; but as it is the result of personal experience, what I have to relate may be of some use as confirmatory of statements of others. Mr. Peal would not be able accurately to estimate direction, unless the sense of hearing-the capacity to receive sound-was precisely equal and similar in each ear. A greater sensibility in one than in the other, would incline him to the right, or the left, as the case might be.

Few people know that they may be partially deaf on one side, and yet not perceive it, just as some are right- or left-eyed, without knowing it. A good test is a watch slid along a two-foot rule, the end of wbich touches the cheek. The watch is moved away, inch by inch, till the ticking is no longer audible-if the distance is the same on both sides, the sensibility of each ear is of course equal.

I shall not forget my horror when my medical man, applying this test, showed that while with my right ear I could distinguish the ticks far beyond the 2 feet, my left ear was impervious to the sound until within an inch or two of the watch. Hearing on that side has now totally ceased, and the result is $I$ have not the slightest idea of the direction of sound. I can hear certain sounds (for I am becoming deaf on the right side also), but to whichever side I incline my right ear, from thence do I fancy the sound to come.

To me, with my passion for ornithology, it is a terrible deprivation. In my youth my senses were intensely acute. I could instantly detect and proceed to the faintest note uttered by a bird in the forest. Now the loudest call only puzzles me the more. But I was a long time before I found this out. I fancied more than once that the bird I was pursuing had powers of ventriloquism; then that there were two or more, calling from different directions. As soon, however, as I found, as before stated, that I was deaf on one side, I began experimenting on niyself, and quickly arrived at the conclusion that in order to estimate direction, both ears needed to be equally sensitive.

A curious instance of correct judgment as to direction and distance of sound is given by one of the South African explorers, Green, I think. He had been much annoyed by a lion which roared round his camp. Taking his rifle and some of his native followers, he went outside in the direction of the sound. Halting, they listened intently, and at the next roar caught the exact direction, and judged the animal to be at (I think) about 300 yards. Carefully levelling his rifle, he fired, and had the satisfaction of hearing the well-known "thud" of the bullet, and a change of note from the midnight serenader. Next morning showed traces of blood, and following up the track they found, and despatched the wounded beast.

Colour-blindness is represented in the other sense, by an inability to distinguish certain sounds. That this is caused by partial deafness, 1 am now pretty well certain. My father-inlaw, who never knew he was deaf, never heard the chirrup of the cricket, and I now find I cannot do so. Only last night my wife observed "what a noise the crickets are making," to me there was unbroken silence as regarded outward sounds. I only heard the continuous "buzzing" that sounds in my head, augmented by the regular " thud thud" of my pulse.

I have a clock, the hours of which strike on a sweet-toned, metallic bell. If my right ear is turned towards it, at a moderate distance, I hear the ringing note; if turned away, I am only conscious of the "burr" of the works, and a dull "thud, thud," denoting the stroke. Why do I hear the "burr," which is not so loud, or clear, as the metallic "ting"? That wave of sound seems to pass by and not affect me; it is like the sharp note of the cricket.

The cause why certain sounds are inaudible to certain ears is a subject well worthy of investigation, as bearing on the placing of sentries or outposts at night, in time of war; also for sportsmen hunting large dangerous game. E. L. LAYARD

British Consulate, Noumea, New Caledonia, A pril 7

\section{Jamaica Petrel}

Mr, D, MORRIS asks (NATURE, vol. xxv, p. I5I) for some clue to the locality and general character of the nesting-places of petrels. As 1 fail to find any reply in your pages up to January 19, I venture to send my mite by way of response to Mr. Morris.

Most of the petrels (Asstrelata), the Storm Petrels (ThalassiAroma) and the Sheerwaters (Puffinus) breed in holes in the ground, excavated by themselves; sometimes on small islands, at other times on high mountains, at considerable dis. tances from the sea. Their novements to and fro are almost always performed at night, and as they are capable of a very rapid flight, a distance of fifteen or twenty miles is quickiy traversed.

Here, in New Caledonia, the well-known AEstrelata mollis breeds on the summit of Mont Mou (about 4000 feet) in JanuaryMarch, in great numbers, laying one white egg, as usual. I am informed that in some places the ground is honeycombed $\mathrm{u}$ ith their burrows. I am also told that during the non-breeding season numbers come to roost in their old holes.

The larger, AE. rostrata, Peall, nests in similar places, but at a much less elevation, on the Island of Uen, the most southern portion of New Caledonia, and hardly divided from it by the celebrated Wodin passage.

Other species are said to frequent other mountains in the interior, but I have no personal knowledge of them.

In Fiji I obtained Puffinus ungax, which bred far away in the mountainous interior, and there are other true petrels which do the same.

Vast numbers of various petrels and shearwaters are found in these seas, and I fancy all burrow, more or less, in the earth, to lay their eggs. Of the "Great Grey Petrel" (Adamastor cinerea) Capt. Hutton says, that it "burrows horizontally into the wet, peaty earth" (of Kerguelen's land) "from two to eighteen

That the "Jamaica Petrel" resorts to the Blue Mountain range of Jamaica, for the purposes of breeding, I have not the smallest doubt, and if the holes are examined at the right time of year, I feel sure eggs will be found. The birds probably do $u=e$ the holes as resting places, during certain periods of the year. They do not, however, lay their eggs at sea! and sooner or later Mr. Morris may be sure of finding eggs, though he may not find much of a nest.

The breeding of the Mutton Bird (Puffinus brevicaudus) on many of the Australian islands has been often described. Its burrows render walking positively dangerous. If Mr. Morris can refer to Gould's "Birds of Australia," he will find much information on this head.

E. L. LAYARD

British Consulate, Noumea, April ro

The Regnard Incandescent Lamp.-In reply to several correspondents who find a difficulty in the use of petroleum for this lamp, we think they may be more successful with benzoline.

\section{DOUBLE STARS}

II.

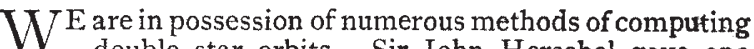
double star orbits. Sir John Herschel gave one of the first solutions of this problem, and his method has been used more than any other up to this, and so far from becoming obsolete, it is yearly gaining ground at the cost of the methods that have been proposed elsewhere. It starts with the construction of the orbit, which the companion appears to describe round the main star. It is clear that as the planes of the orbits may be inclined in every direction in space, we see only the projection of the real orbits on the heavens, but this, as well as every other projection of an ellipse on a plane surface, is another ellipse, though the main star does no longer appear situated in the focus. Five points determine an ellipse, if we therefore possess five complete observations, we can determine the apparent ellipse. Now the observations are not perfectly accurate, but the calculus of probabilities furnishes us with means to ascertain the most probable ellipse from a great number of observations, to which different weight may be attributed, according to their reliability, as far as known. But at Herschel's time, though the angles had been fairly observed, the measurement of these minute distances was still in its infancy. He, in consequence, threw them away, and computed distances by aid of the Keplerian law referred to above, from the angular velocities, concluded from a comparison of observations separated by moderate intervals. He improved the angles in the following way :On a paper neatly divided into squares, he lays down a

\footnotetext{
I Continued from p. 155.
} 
point for every observed angle of position, the epoch in years and decimals being measured as an abscissa along the horizontal lines, and the angle in degrees as an ordinate along the vertical ones. A series of points are thus obtained, which, if the observations were exact, would necessarily admit of a regular curve being drawn through them, whose nature is of course determined by the laws of elliptic motion, and one of whose essential characters is to have within those limits of the abscissa, which correspond to a whole period of revolution (that is, to a difference of 360 units in the ordinates), in some cases two, in some four, points of contrary fiexure, but never more than the latter, nor fewer than the former, and to have, moreover, in all its points, a peculiarly graceful and Aowing outline. The errors of observation, however, prevent the drawing of such a curve through all the points. It must be drawn with a free but careful hand, not through, but among the points, and so that it shall deviate less from every point, according as it is more or less reliable. Now after Herschel's time the accuracy of the observed distances has wonderfully improved, and we are therefore able to draw another curve representing the distances as ordinates, which then ought to agree with those deduced from the angles, and the angles ought to agree with those deducible by aid of integral calculus from the distances. The curves must be varied till they thus mutually support each other, and then we may construct any number of points of the apparent orbit by reading off the angles and distances for the corresponding eposhs on the curves, and if we find the arc described sufficiently extensive, the apparent ellipse is simply drawn as nearly as possible through them. From the apparent orbit the elements of the real orbit, described in space, are then determined. These are seven in number:-

a. The major semi-axis, expressed in seconds of arc, i.e. the angle under which their mean mutual distance would appear if placed perpendicular to the line of vision, $i e$. the straight line joining us with the star.

$\epsilon$. The eccentricity of the real ellipse.

$\gamma$. The inclination of the plane of the real orbit to the plane perpendicular upon the line of vision.

$\Omega$. The node, i.e. the angle of position of the line in which the plane of the orbit intersects the plane perpendicular upon the line of vision.

$\pi$. The longitude of the projected peri-astron, i.e. the angle of position of the companion at the epoch of its actual nearest approach to the main star.

T. The epoch, when the nearest approach in space occurs.

$P$. The period of revolution, i.e. the time it takes the companion to complete an entire revolution round the main star.

It is impossible to say what part of the orbit is inclined towards us, and what is removed from us, ${ }^{1}$ we cannot therefore, distinguish between an ascending and a descending note.

Both before and after Herschel's investigations, several methods of calculating double-star orbits have been proposed. Savary, at Arago's request, was the first who gave an analytical determination of an orbit from five points. He also proposed a method, subsequently improved by Encke, for calculating the seven elements of the real orbit from four complete observations. These furnish eight co-ordinates corresponding to known epochs, and as only seven quantities, the elements are sought, the problem is over-determined. It has to be solved with different values of one of the data, in order that all the other data may be represented. The case is, at the time it generally was possible to obtain but three complete positions from the discussion of modern observations, while the fourth, depending upon Herschel's measures,

I This could be ascertained if we could at any time observe the speed in miles per second with which the companion approaches to or recedes from us in the line of vision. Spectroscopists are making some progress in similar researches, but their apparatus are not as yet sufficient for our purpose. gives seldom even an approximate value of the distance. After that, a longer series of angles had become available. Klinkerfues proposed to determine at once the seven elements from six observed angles, and at least one distance. The dimensions of the orbit could evidently not be obtained without the aid of observed dimensions, though all the other elements can be derived from the angles. It sometimes occurs that the companion moves in an orbit, the edge of which is turned towards us. The inclination is then about ninety degrees, and all the angles are nearly equal to, or half a circumference different from the longitude of the node. We are then obliged to make more extensive use of the distances. Several analytical methods have been adopted to this contingency, which has actually occurred in a few cases. It seldom happens that an orbit founded upon a few positions, even if these are distributed over a great part of the arc described, is the most accurate that could possibly be deduced from all the observations at our disposal. It is therefore advisable to finally correct the elements according to the rules of the calculus of probabilities, so that the errors ultimately left behind in the representation of the observed places, may, taking into account their relative accuracy and their different peculiarities, be as small as possible. In this country, Hind, one of the greatest authorities on anything relating to double stars. has made most extensive calculations of this nature.

The number of those double stars whose orbits can be computed is limited to between thirty and forty. The time during which micrometric measures have been made is so short, that one but seldom can make anything like a correct guess of what the whole orbit may turn out to be like, when the period is above three hundred years ; and even so, the peri-astron passage must happen to fall within a hundred years from now ; that is, if the eccentricity is not unusually small. The eccentricity of these orbits is generally large, and it appears to be larger the greater the period and the greater the axis is. Now in the cases-by far the most frequent - where the measures do not embrace a larger part of the orbit, we represent the motion of the companion by formula, deduced in accordance with the proportionality of the areas with the times. These formulæ suffice to foretell the co-ordinates for some few years to come, and they are interesting in other respects, too. Thus, if the distance of a double star from the solar system were known to us, we would also know the dimensions of the orbit in miles, and then we could calculate its mass by aid of Kepler's laws. Now their distances from us are not known, but if we assume the mass to be on an average e.g. about three times the mass of the sun, then we obtain-if we make the further assumption that the distance actually measured in seconds of arc between the components is in an average of a very great number of stars equal to their mean distance from each other-from the period of revolution (concluded from the variation of the angle) through the inverse calculation their distance froin us. Now these hypotheses can only be used in discussing the results of an average of a great number ot systems that have certain characters in common, e.g. brightness, colour, or distance. I am at present engaged with such researches, from which I hope to arrive at interesting results.

Most of the double stars, that so far have been found to revolve, are close and more or less difficult objects. Few of them are more than six and a half seconds of arc asunder. The components are in most cases of about the same brightness, but the companion is, on the whole, smaller, the greater the distance.

There are two kinds of revolving double stars. The first of them consists of bodies whose colours are strictly identical, whereas the second consists of bodies whose colour is generally complementary. The principal star in both cases is white or yellow-white stars preponderating in the first case, yellows in the second. In systems of 
the latter kind, the companion is generally bluish, and the number of blue companions increases rapidly with the distance, so that the close pairs are generally of the same, more or less white colour, and not very different in magnitude, whereas the wide pairs are of complementary colours, and the companion much fainter than the primary. We see then that the brightness is, on the whole, more different, the more different the colour is-a circumstance first pointed out by Struve. Now it is well known that the colour of a solid or liquid cooling body passes from white through yellow to red, and these are the colours in which the single stars, without exception, appear to us, whereas the blue and purple stars are found only as small companions to brighter stars. Holden, in Washington, has suggested that these colours are due to absorptive atmospheres-an opinion, the decision of which we leave to spectroscopists. Pickering, in Cambridge, U.S., has shown, from the absence of polarisation, that they do not shine with reflected light.

The last remarks remind us of the planets, that, no doubt, revolve round those distant suns, and derive from them their light and heat. When we reflect upon the complicated nature of the orbits, which the planets and comets describe round our sun, we get some idea of how remarkable must be the nature of those curves that planets describe round double suns, compared to which the motion of our moon is simple, and easily understood. Perhaps the same comets that disappear to our gaze, leaving the sphere of attraction of the sun, are attracted, and for a time become members of those wonderful systems. Our mathematics do not suffice for solving the problems that are thus suggested, but it is not unlikely that, unless situated very close to one or other of the suns-so close that the other appears not much larger than a star, though comparable in brightness to the nearest sun -the planets are whirled out in the cold space by the gravitational influence of the other sun, as very likely many a minor planet was ejected from the solar system under the influence of Jupiter. What must be the nature of those worlds illuminated by two different suns, one yellow and another purple? Now rises the one, and all is clothed in yellow, now the other, and illuminated from complementary sources, every object appears in its natural colour. Then sets the yellow sun, and what must be the diversity of the effects as it approaches the horizon! And behold nature puts on a purple mantle. Then also that sun sets, and in the darkness of night, though there is seldom night where there are two suns, the starry heavens are seen much the same there as here, except perhaps for moons reflecting light from the differently-coloured suns. But stars that seem large to us are hardly visible there, while our sun is perceived in the telescopes of the mysterious beings that inhabit those strange globes as only a faint star, and metaphysicians there prove from a priori considerations to their attentive pupils, that no life could bask in the feeble glare of a single sun-how all would famish but for the opposite effects of the two suns. And no doubt! life there is heightened, and the wonders of nature are unravelled by aid of and under the influence of the energy of several suns, more highly developed science, seeing how glorious is the creation on this poor orb, that is kept alive by but one. Not only the play of colour must there be more varied than here, but phenomena of which we have not the faintest idea, must be produced also through the action of heat, electricity, and magnetism. ${ }^{1}$ Indeed, upon subjects like these, science gives no information, and we may therefore give our fancy free reins. Oh! that we did possess the power of appreciating these things like the divine Milton, when in truly prophetic strain he wrote :-

$$
\begin{aligned}
& \text { "Other suns, perhaps, } \\
& \text { With their attendant moon", thou wilt descry, }
\end{aligned}
$$

\footnotetext{
The tides upon the planets caused by the two suns must be very interest-
}

Communicating male and female light,

Which two great sexes animate the world,

Stored in each orb, perhaps, with some that live ;

For such vast room in nature unpossess'd,

By living soul, desert and desolate,

Only to shine, yet scarce to contribute

Each orb a glimpse of light, conveyed so far

Down to this habitable, which returns

Light back to them, is obvious to dispute."

\section{W. DOBERCK}

\section{ON THE PHOTOGRAPHIC SPECTRUM OF COMET (WELLS) $I_{.,} 1882^{1}$}

O

$\mathrm{N}$ May $3 \mathrm{I}$ I obtained a photograph of the spectrum of this comet, with an exposure of one hour and a quarter. On the same plate I took a spectrum of $a$ Ursæ majoris for comparison. The comet's spectrum on the plate consists of a strong continuous spectrum extending from about $F$ to a little beyond $H$. I am not able to distinguish any of the Fraunhofer lines in this continuous spectrum. The slit was rather more open than was the case in photographing the spectrum of the comet of last year; this would make these lines less distinct, but the lines $G$ and $H$ are well seen in the star's spectrum taken under the same conditions. We may therefore conclude that the part of the comet's original light which gives a continuous spectrum is much stronger relatively to the reflected solar light in this comet, than was the case in the comet of last year, and for this reason the Fraunhofer lines are not distinguishable.

Observations of the visible spectrum had already shown that the comet differs remarkably from the hydrocarbon type common to all the comets, some twenty, which have appeared since spectrum analysis has been applied to these bodies.

The photographic spectrum shows, as was to be expected, that this essential difference of spectrum exists also in the more refrangible region. The very strong ultra-violet group assigned to cyanogen is not to be seen on the plate, and the bright groups between $\mathrm{G}$ and $h$, and between $h$ and $\mathrm{H}$ do not appear to be present.

The head of the comet was in sharp focus upon the slit, and the continuous spectrum with defined edges corresponds to the nucleus which in this comet was very distinct. In this continuous spectrum at least five separate places of greater brightness are seen, which very probably represent groups of bright lines, though they are not sufficiently distinct in the photograph to admit of resolution. That this interpretation is correct, seems probable, from the circumstance that these groups, as shown in the diagram, project beyond the strong continuous spectrum on one side. This side corresponds to where the light of the coma, on the side of the nucleus next the sun, falls upon the slit. We learn, therefore, that the light of this part of the coma consists for the most part in this part of the spectrum of these groups, as here on the plate only an exceedingly faint continuous spectrum can be seen.

It is not possible to measure with any useful accuracy the beginnings and endings of the groups, as they are too faint at these points. Measures as accurate as the circumstances would permit have been taken of the brightest parts of the groups. The wave-lengths of these brightest $\lambda 4253$ $\lambda 4412$

parts are : $\lambda 4507$

$\lambda 4634$ $\lambda 4769$

In the visible spectrum the bright lines of sodium

ing. Of course they also produce tides upon each other, and their spots present no doubt most peculiar features. It is not unlikely that in some cases the phenomena presented by new as well as by some variable stars are to be explained as the effect of tides caused by darker companions.

I Substance of note read befire the Royal Society, June 15,1882 . 